\title{
Gear Fault Diagnosis Based on VMD Sample Entropy and Discrete Hopfield Neural Network
}

\author{
Jiakai Ding, ${ }^{1,2}$ Dongming Xiao $\mathbb{D}^{1,2}$ Liangpei Huang, ${ }^{1}$ and Xuejun $\mathrm{Li}^{2}$ \\ ${ }^{1}$ Hunan Provincial Key Laboratory of Health Maintenance for Mechanical Equipment, \\ Hunan University of Science and Technology, Xiangtan 411201, China \\ ${ }^{2}$ School of Mechatronics Engineering, Foshan University, Foshan 528225, China \\ Correspondence should be addressed to Dongming Xiao; dongming.xiao@outlook.com
}

Received 17 September 2020; Revised 28 October 2020; Accepted 30 November 2020; Published 18 December 2020

Academic Editor: Antonio Forcina

Copyright (C) 2020 Jiakai Ding et al. This is an open access article distributed under the Creative Commons Attribution License, which permits unrestricted use, distribution, and reproduction in any medium, provided the original work is properly cited.

\begin{abstract}
The gear fault signal has some defects such as nonstationary nonlinearity. In order to increase the operating life of the gear, the gear operation is monitored. A gear fault diagnosis method based on variational mode decomposition (VMD) sample entropy and discrete Hopfield neural network (DHNN) is proposed. Firstly, the optimal VMD decomposition number is selected by the instantaneous frequency mean value. Then, the sample entropy value of each intrinsic mode function (IMF) is extracted to form the gear feature vectors. The gear feature vectors are coded and used as the memory prototype and memory starting point of DHNN, respectively. Finally, the coding vector is input into DHNN to realize fault pattern recognition. The newly defined coding rules have a significant impact on the accuracy of gear fault diagnosis. Driven by self-associative memory, the coding of gear fault is accurately classified by DHNN. The superiority of the VMD-DHNN method in gear fault diagnosis is verified by comparing with an advanced signal processing algorithm. The results show that the accuracy based on VMD sample entropy and DHNN is $91.67 \%$ of the gear fault diagnosis method. The experimental results show that the VMD method is better than the complete ensemble empirical mode decomposition with adaptive noise (CEEMDAN) and empirical mode decomposition (EMD), and the effect of it in the diagnosis of gear fault diagnosis is emphasized.
\end{abstract}

\section{Introduction}

Gears are widely used in modern industrial machines and play a key role. When the gear is damaged, the transmission machinery will cause huge economic losses. The corresponding vibration signal will be generated when the gear runs under normal, wear, cracked, and broken teeth, which contains abundant fault information [1]. Therefore, it plays an important role to monitor the running state of the gear, which can be detected and replaced when the early weak fault occurs.

The most mature gear monitoring technology is based on the vibration signal. When the gear is damaged, the corresponding vibration signal will change. Therefore, it is only necessary to collect the vibration signal of gear under the corresponding fault state and extract the corresponding feature, and then, the fault diagnosis of gear can be carried out. The innovation of gear fault diagnosis technology is based on the signal method. Traditional gear fault diagnosis methods include time-frequency domain analysis. Classical signal processing methods such as wavelet packet transform (WPT), Hilbert-Huang transform (HHT), and short-time Fourier transform (STFT) have been widely used in the field of fault diagnosis [2]. Mohammed et al. [3] utilized the vibration signal for gear fault diagnosis. Rafiee [4] utilized autocorrelation of continuous wavelet coefficients (CWCs) for gear fault diagnosis. These traditional time-frequency transforms are still insufficient in time-frequency resolution. Empirical mode decomposition (EMD), local mode decomposition (LMD), and other modern signal processing methods have also been applied [5-7]. Rafiee et al. [8] introduced an automatic feature extraction system for gear and bearing fault diagnosis using wavelet-based signal processing. However, EMD and LMD methods have 
problems such as mode-mixing and endpoint effect $[9,10]$. In order to effectively solve the mode-mixing and endpoint effect of the EMD and the LMD, variational mode decomposition (VMD) came into being [11]. VMD overcomes the shortcomings of the traditional signal adaptive decomposition methods of EMD and LMD, which converts the signal decomposition into a variational problem and solves the signal adaptive decomposition by seeking the optimal solution of the problem. However, the parameter combination of the penalty factors and the number of decomposition should be determined before VMD decomposition, which brings great difficulties to the accurate decomposition of signals. Some researchers used an optimization algorithm to optimize the parameter combination of the penalty factors and the number of decomposition in the VMD decomposition process. The genetic mutation particle swarm optimization (GMPSO) algorithm to optimize the VMD algorithm parameters is utilized by Ding $[12,13]$. Experimental results show that he GMPSO-VMD algorithm has a good decomposition effect on the gear fault signal. The optimal adaptive VMD decomposition algorithm can adaptively determine the penalty factor and decomposition number in the VMD algorithm according to the time-frequency characteristics of different fault signals [14]. A variational mode decomposition method based on a cuckoo search algorithm to adjust the changes in internal parameters in VMD decomposition is utilized by Yan and Jia [15], and the multicomponent signal could be adaptively decomposed into a subsignal superposition of inherent mode function. The VMD adaptive decomposition algorithm [16] can be realized by adaptively adjusting the parameters of vibration signals of rotating machinery under VMD decomposition, such as the optimal number of mode decomposition and frequency bandwidth control.

The intrinsic mode functions (IMFs) of the original fault signal were decomposed by the VMD algorithm, the IMFs contain abundant characteristic parameters, and different characteristic parameters represent different physical meanings. Therefore, selecting more effective feature parameters can increase the recognition accuracy for subsequent fault diagnoses. The vibration signal of rotating machinery is usually nonlinear and nonstationary, which makes it very difficult to extract fault features. Entropy value can be used as a very effective parameter to extract fault features [17]. In order to improve the fault identification accuracy of rolling bearing, Chen applied the rapid sample entropy [18] and the improved multiscale amplitude-aware permutation entropy (IMAAPE) [19] to the feature extraction of rolling bearing diagnosis signal and achieved good results. A milling flutter detection method based on VMD and energy entropy is proposed by Liu et al. [20]. In order to automatically detect the flutter frequency band better, a flutter detection method based on energy entropy was proposed. The energy entropy value is taken as the fault feature parameter to extract, and it is verified that the energy entropy value can effectively express the flutter characteristics. Therefore, according to the advantages of simple sample entropy calculation and fast calculation speed, this paper selects it to extract the vibration signal feature of gear fault.

The feature vectors after feature extraction can be used as the input vectors in the fault diagnosis model. As a classifier, support vector machine (SVM) has been studied by researchers for a long time. VMD is used to extract features from signals, and SVM is used for fault diagnosis. The results show that, under complex conditions, the proposed method can also perform fault diagnosis more accurately [21, 22]. However, in the process of SVM classification, kernel functions need to be solved according to specific problems and are not self-adaptive. In addition, there are many deep learning algorithms for fault diagnosis research, such as deep belief network (DBN) [23], long short-term memory (LSTM), deep self-encoder (DSE), deep convolution neural network (DCNN), and other deep learning algorithms [24-28] in recent years. However, the training time of the deep learning algorithm is too long to make fault diagnosis in time. The discrete Hopfield neural network (DHNN) is a recursive neural network, which was first proposed by JJ Hopfield [29]. Its operating mode is a binary system, and the value of the network node is only 1 or -1 . It is a neural network model that can simulate human memory. In the field of mechanical fault diagnosis, DHNN has not been applied to gear fault diagnosis. So it is a new attempt to apply DHNN to gear fault diagnosis.

Based on the shortcomings of the EMD and the LMD, the optimized VMD is utilized to decompose the gear fault vibration signal. The sample entropy value is extracted as the characteristic parameter. Because the sample entropy is sensitive to the change of signal chaos, the sample entropy as the signal feature parameter is extracted by this paper. Based on the above literature on deep neural network algorithms, it is concluded that the deep neural network algorithm has too long training time in fault diagnosis and cannot quickly make fault diagnosis. Therefore, this paper synthesizes the shortcomings of the above literature. In order to quickly and accurately diagnose faults, a gear fault diagnosis based on VMD sample entropy and DHNN is proposed.

\section{Experimental System and Methods}

2.1. Experimental System. This section verifies the gear fault diagnosis capability of the VMD-DHNN method under different working conditions. This method mainly analyzes the fault signals collected from the gear experimental test rig. The gear experimental test rig is shown in Figure 1. The active and slave gears are all bevel gears, and the vibration acceleration signals from the gearbox are collected by using B\&K data collector. Meanwhile, the sampling frequency and sampling time of the experiment were $8192 \mathrm{~Hz}$ and $0.25 \mathrm{~s}$, respectively. The test rig includes an electromagnetic speed control motor controller, a three-phase asynchronous motor, two couplings, a reducer, an acceleration sensor, a B\&K data acquisition analyzer, and a laptop. The gear fault parts are shown in Figure 2.

\subsection{VMD Sample Entropy and DHNN Fault Diagnosis} Method. As mentioned in Introduction, the VMD is selected in this paper to avoid the endpoint effect and mode confusion of the EMD and the LMD. The VMD has the advantage of high accuracy of the center frequency of each IMF and fast calculation speed, while DHNN has the 


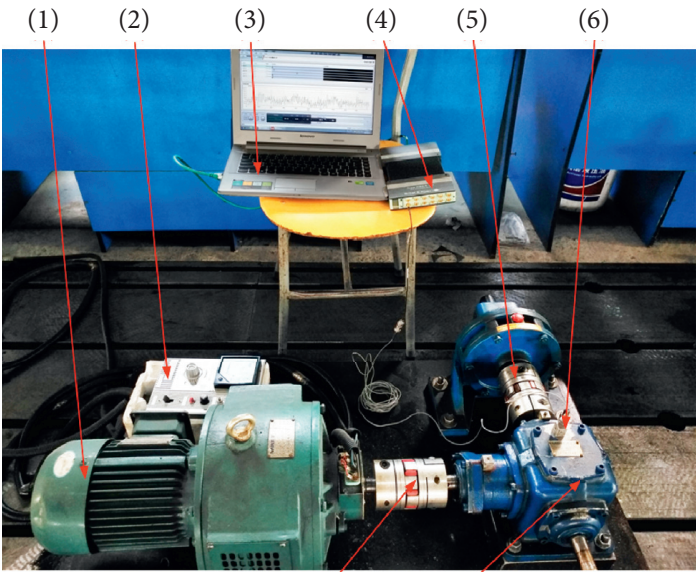

$(8)$

(7)

FIGURE 1: Gear experimental test rig: (1) three-phase induction motor; (2) electromagnetic speed control motor controller; (3) laptop; (4) B\&K data acquisition analyzer; (5) coupling; (6) acceleration sensor; (7) reducer; (8) coupling.

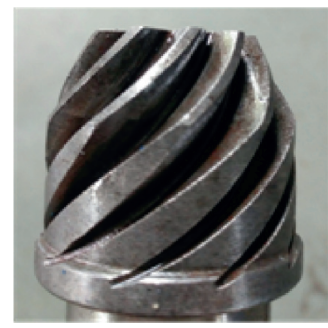

(a)

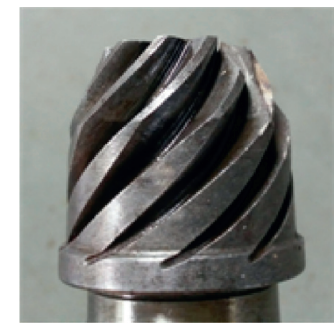

(b)

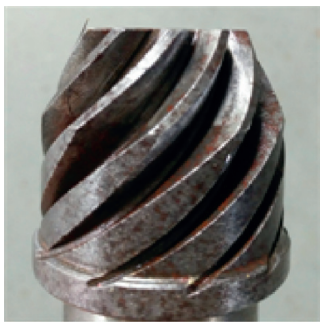

(c)

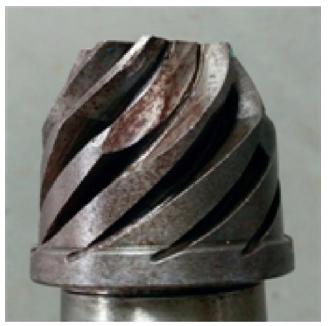

(d)

FIgURE 2: Gear fault parts: (a) normal gear; (b) gear with tooth crack; (c) gear with tooth wear; (d) gear with tooth break.

advantage of associative memory to speed up the calculation speed and improve the accuracy. The effectiveness of the method is verified by the measured gear fault diagnosis experiment. The implementation steps of the VMD-DHNN method are shown in Figure 3.

The detailed steps of the VMD-DHNN method are as follows:

(i) Step 1. The optimal number of the VMD decomposition algorithm is selected.

(ii) Step 2. The optimized VMD algorithm is utilized to decompose the gear fault signal.

(iii) Step 3. The sample entropy value is extracted from each IMF, which form the feature vector of gear fault.

(iv) Step 4. The gear fault feature vectors are encoded.

(v) Step 5. The encoded fault feature vectors are input to DHNN for gear fault diagnosis.

(vi) Step 6. Output results.

\section{Gear Fault Feature Extraction of VMD Sample Entropy}

3.1. VMD Method. Dragomiretskiy and Zosso proposed the VMD algorithm [11] in 2014. The original gear signal $f(t)$ is decomposed into $k$ IMFs $x_{k}(t)$ by the VMD algorithm. The core of VMD is to construct an L2 norm equation, whose formula is expressed as

$$
\begin{gathered}
\min _{\left\{u_{k}\right\},\left\{\omega_{k}\right\}}\left\{\sum_{k}\left\|\partial_{t}\left[\left(\delta(t)+\frac{j}{\pi t}\right) \times x_{k}(t)\right] e^{-j \omega_{k} t}\right\|_{2}^{2}\right\}, \\
\text { s.t. } \sum_{k=1} u_{k}=f(t), \\
L\left(\left\{x_{k}\right\},\left\{\omega_{k}\right\},\{\lambda\}\right)= \\
\alpha \sum_{k}\left\|\partial_{t}\left[\left(\delta(t)+\frac{j}{\pi t}\right) \times x_{k}(t)\right] e^{-j \omega_{k}}\right\|_{2}^{2} \\
+\left\|f(t)-\sum_{k} x_{k}(t)\right\|_{2}^{2}+\langle\lambda(t), f(t) \\
\left.-\sum_{k} x_{k}(t)\right\rangle,
\end{gathered}
$$

where $\omega_{k}$ is the center frequency of the IMFs and $\partial_{t}$ is the differential symbol.

The alternate direction method of multipliers (ADMM) is used to calculate equation (2), and the optimal solution is expressed as

$$
\begin{aligned}
\widehat{x}_{k}^{n+1}(\omega) & =\frac{\widehat{f}(\omega)-\sum_{i \neq k} \widehat{x}_{i}(\omega)+\left(\widehat{\lambda}_{i}(\omega) / 2\right)}{1+2 \alpha\left(\omega-\omega_{k}\right)^{2}}, \\
\widehat{\omega}_{k}^{n+1} & =\frac{\int_{0}^{\infty} \omega|u(\omega)|^{2} \mathrm{~d} \omega}{\int_{0}^{\infty}\left|u_{k}(\omega)\right|^{2} \mathrm{~d} \omega},
\end{aligned}
$$




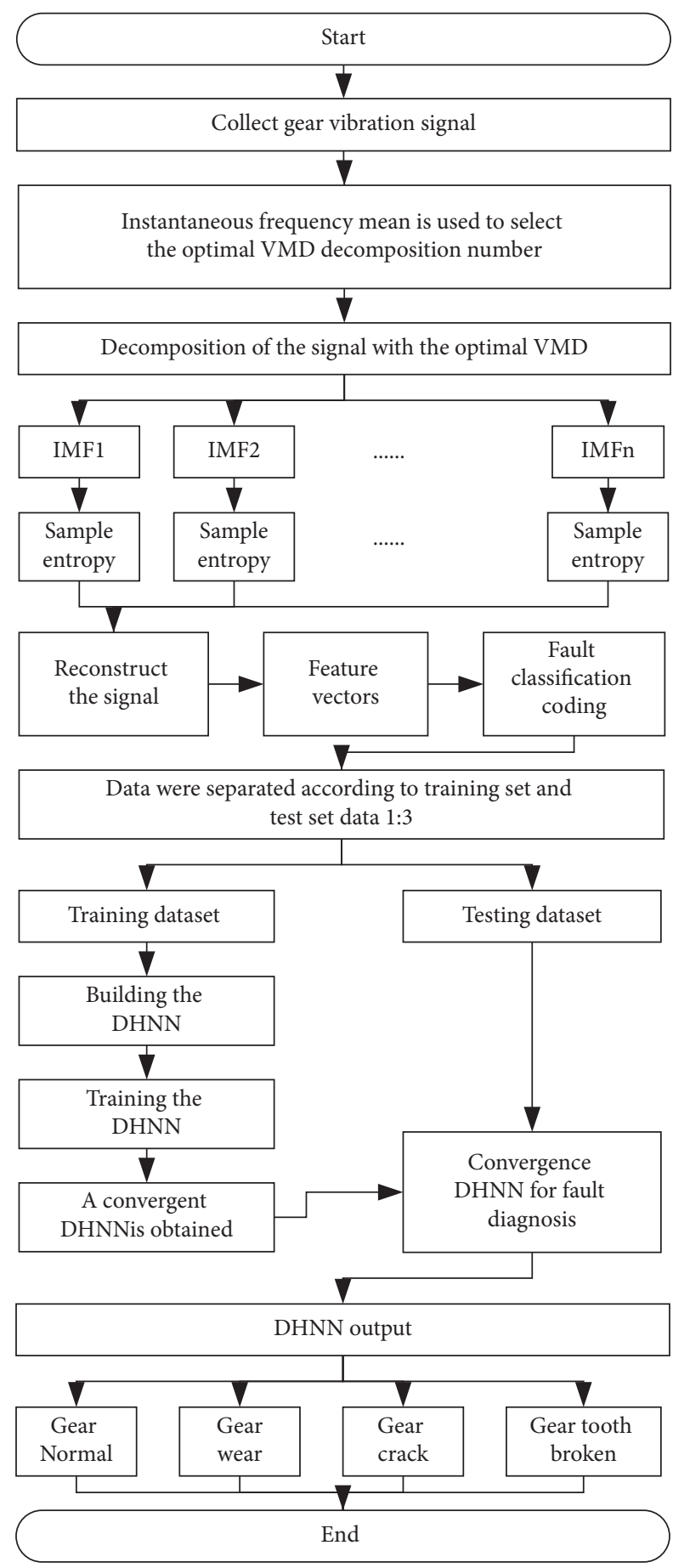

FIgUre 3: The flow chart of VMD sample entropy and DHNN gear fault diagnosis.

where $\hat{f}(\omega), \widehat{x}_{i}(\omega)$, and $\hat{\lambda}(\omega)$ represent the Fourier transform of $f(\omega), x_{i}(\omega)$, and $\lambda(\omega)$, respectively, and $\varepsilon$ represents the discriminant accuracy.

According to reference [30], the optimal decomposition number of the VMD is selected through the change in instantaneous frequency mean value. This paper also selects the optimal decomposition number of the VMD by the same method. According to literature [11], the penalty factor $\alpha$ and discriminant accuracy $\varepsilon$ of the VMD algorithm are 2000 and 10-7, respectively. After repeated experimental analysis, the optimal decomposition number $k=4$.

3.2. Sample Entropy Method. Sample entropy is an indicator to measure the complexity of time series signals. The higher the sample entropy value of the signal, the more complex the signal. The sample entropy is calculated as follows:

(i) Step 1. A vector sequence with dimension $m$ by ordinal number is formed, $X_{m}(1), \ldots, X_{m}(N-$ $m+1)$, where $x_{m}(i)=\left(x_{m}(i), x_{m}(i+1), \ldots, x_{m}(i+\right.$ $m-1)$ ). These vectors represent $m$ consecutive values of $x$ starting at the point $i$.

(ii) Step 2. The distance between vector $X_{m}(i)$ and $X_{m}(j)$ is defined, and $d\left[X_{m}(i), X_{m}(j)\right]$ is the absolute value of the maximum difference between the corresponding elements, that is,

$d\left[X_{m}(i), X_{m}(j)\right]=\max _{k=0,1, \ldots, m-1}(|x(i+k)-x(j+k)|)$.

(iii) Step 3. For a given $X_{m}(i)$, the number of $j(1 \leq j \leq N-m, j \neq i)$ is counted whose distance between $X_{m}(i)$ and $X_{m}(j)$ is less than or equal to threshold $r$, and it is called $B_{i}$. For $1 \leq i \leq N-m$, $B_{i}^{m}(r)$ is defined as

$$
B_{i}^{m}(r)=\frac{B_{i}}{N-m+1}
$$

(iv) Step 4. The average value of the $B_{i}^{m}(r)$ is defined as

$$
B^{m}(r)=\frac{1}{N-m} \sum_{i=1}^{N-m} B_{i}^{m}(r)
$$

(v) Step 5. The dimension to $m+1$ is increased, the number of $j(1 \leq j \leq N-m, j \neq i)$ is counted whose distance between $X_{m+1}(i)$ and $X_{m+1}(j)$ is less than or equal to threshold $r$, and it is called as $A_{i}$. $A_{i}^{m}(r)$ and is defined as

$$
A_{i}^{m}(r)=\frac{A_{i}}{N-m+1} \text {. }
$$

(vi) Step 6. The average value of the $A_{i}^{m}(r)$ is defined as

$$
A^{m}(r)=\frac{1}{N-m} \sum_{i=1}^{N-m} A_{i}^{m}(r) .
$$

So the sample entropy is defined as

$$
\operatorname{SampEn}(m, r, N)=-\ln \left[\frac{A^{m}(r)}{B^{m}(r)}\right] .
$$


The 16 sets of data in the four states were separately decomposed by VMD. Through the previous analysis, the original gear fault signal is decomposed into 4 IMFs, and then, the sample entropy value of each IMF is extracted to form the gear fault feature vector. G1 represents the normal gear, G2 represents the gear with tooth wear, G3 represents the gear with tooth crack, and G4 represents the gear with tooth break. SampEn1 represents the sample entropy value of the component IMF1, SampEn2 represents the sample entropy value of the component IMF2, SampEn3 represents the sample entropy value of the component IMF3, and SampEn4 represents the sample entropy value of the component IMF4. Table 1 shows the sample entropy values of the IMFs.

In order to obtain a better classification effect, it is preliminarily classified from the size range of the overall sample entropy. The distribution of the size range of the overall sample entropy is shown in Table 2.

Then, the entropy value range of the samples of normal gear, gear with tooth wear, gear with tooth crack, and gear with tooth break is divided from SE1-SE4 (see Table 3 for details).

\section{DHNN Fault Diagnosis Model}

4.1. DHNN Method. This paper attempts to find a new method of gear fault diagnosis and establishes a new diagnosis model for gear fault diagnosis. Therefore, a new method of discrete Hopfield neural network (DHNN) was proposed to diagnose the entropy characteristics of gear fault samples, so as to solve the problems of low fault diagnosis accuracy and slow diagnosis speed of traditional methods. Through testing the established model, the results showed that the algorithm had better detection and diagnosis results.

Due to its network structure and activation function settings, DHNN has the functions of associative memory and nonlinear mapping of information, classification, and recognition. It has been widely used in the field of fault diagnosis.

After the VMD decomposition of the gear vibration signal, the sample entropy value is extracted from each IMF to form the feature vectors. Then, it encodes the feature vectors as the memory prototype and memory starting point of the associative memory, respectively. Then, the coding vectors are input to DHNN for fault diagnosis.

The design steps of this fault diagnosis model mainly include the following eight steps, as shown in Figure 4.

4.2. Structure of DHNN. The DHNN is a binary input and output binary neural network. Taking the DHNN network structure model composed of 3 neurons as an example, as shown in Figure 5, two layers of neurons are set, but only the first layer of neurons is the actual neurons. The first layer of neurons reads the input signal of layer 0 , and after weighted cumulative summation, and then activates the sgn function to discriminate and outputs the signal to the next step until the network reaches steady state. The structure of DHNN is shown in Figure 6

Layer 0 is only the input layer of the DHNN, and it is not the actual neuron, so layer 0 has no computing power. The layer 1 is the actual neuron, whose function is to sum the product of the input information and the weight coefficient and produces the output information after the processing of the nonlinear function sgn, where the original information $S E_{i}$ is input from layer 1 to layer 0 for operation.

The entropy value of the gear fault sample $S E=\left(S E_{1}, S E_{2}, \ldots, S E_{i}\right)$ and the neuron node state of DHNN are 1 or $-1 ; 1$ means that the neuron is activated, and -1 means that the neuron is inhibited. $h_{i}(t)$ is the weighted cumulant input by the neuron at the moment. As calculated by the following equation,

$$
h_{i}(t)=\sum_{i=1}^{n} \omega_{i j}-q_{i}, \quad j=1,2,3,
$$

where $\omega_{i j}$ is the connection weight between neuron $i$ and neuron $j, q_{i}$ is the threshold of neuron $i$, and then, the next state $y_{i}(t+1)$ of neuron $i$ is calculated as shown in the following equation:

$$
y_{i}(t+1)=\operatorname{sgn}\left(h_{i}(t)\right)= \begin{cases}1, & h_{i}(t) \geq 0, \\ -1, & h_{i}(t)<0 .\end{cases}
$$

Let $y_{i}(t)$ be the output value of neuron $i$ at time $t$, and the network feeds $y_{i}(t)$ back to the input terminal to become the input value of neuron $i$ at the next moment, thus obtaining the output value of the network at the next moment $y_{i}(t+1)$, as shown in equation (12). After a certain number of iterations of the network according to the calculation method shown in equation (12), the network will converge to a steady state. In this case, the output value of the network should be the same as the output value at the previous moment, as shown in equation (13):

$$
\begin{aligned}
& y_{i}(t+1)=\operatorname{sgn}\left(h_{i}(t)\right), \\
& y_{i}(t+1)=y_{i}(t) .
\end{aligned}
$$

As it can be seen from the above equation, the output value of DHNN is only 1 or -1 , where 1 represents that the neural network node is activated, and -1 represents that the neural network node is suppressed.

4.3. DHNNEncoding Rules. Firstly, the standard data set was input into the DHNN for binary coding, and then, the coding vectors were input into the DHNN for training. The state of neurons in DHNN neural network is only 1 and -1 , and the coding of DHNN neural network needs to change the state of neurons. So, it is necessary to code the evaluation index when mapping to the state of the neuron. Coding rule: when greater than or equal to the index value of a certain level, the corresponding neuron state is set to "1;" otherwise, it is set to " -1 ." The four-grade evaluation indexes of gear fault state are encoded as shown in the following table: • represents the neuron state as " 1 ," which means greater than or equal to the ideal evaluation index value of the corresponding grade; otherwise, it is denoted by $\circ$.

The standard vector value of normal gear is defined here as 1 , the standard vector value of gear with tooth wear is 2 , 
TABLE 1: Sample entropy values of each IMF.

\begin{tabular}{|c|c|c|c|c|c|}
\hline State of the gear & Signal sequence & SampEn1 & SampEn2 & SampEn3 & SampEn4 \\
\hline \multirow{4}{*}{ G1 } & 1 & 0.6542 & 0.7433 & 0.8416 & 0.8176 \\
\hline & 2 & 0.7151 & 0.7666 & 0.8174 & 0.7689 \\
\hline & 3 & 0.7224 & 0.7483 & 0.7932 & 0.8600 \\
\hline & 4 & 0.7105 & 0.7698 & 1.1610 & 0.8194 \\
\hline \multirow{4}{*}{ G2 } & 1 & 0.6871 & 0.7919 & 0.9700 & 1.0353 \\
\hline & 2 & 0.6903 & 0.7964 & 0.9545 & 0.9600 \\
\hline & 3 & 0.6847 & 0.8094 & 0.9436 & 0.9389 \\
\hline & 4 & 0.7001 & 0.7999 & 0.8822 & 0.9433 \\
\hline \multirow{4}{*}{ G3 } & 1 & 0.7307 & 0.8161 & 0.9177 & 0.8973 \\
\hline & 2 & 0.7500 & 0.7711 & 0.8560 & 0.8839 \\
\hline & 3 & 0.7377 & 0.8300 & 0.8618 & 0.8748 \\
\hline & 4 & 0.7186 & 0.8232 & 0.8580 & 0.8604 \\
\hline \multirow{4}{*}{ G4 } & 1 & 0.6514 & 0.8357 & 0.9759 & 0.7661 \\
\hline & 2 & 0.6679 & 0.8478 & 1.0348 & 0.8100 \\
\hline & 3 & 0.6622 & 0.8590 & 1.1180 & 0.7844 \\
\hline & 4 & 0.6198 & 0.7533 & 0.9809 & 0.8218 \\
\hline
\end{tabular}

TABLE 2: Distribution of the size range and the mean value of the overall sample entropy value.

\begin{tabular}{|c|c|c|c|c|}
\hline Sample entropy & SampEn1 & SampEn2 & SampEn3 & SampEn4 \\
\hline Numerical range & $0.65-0.75$ & $0.74-0.86$ & $0.79-1.03$ & $0.76-0.96$ \\
\hline Mean value of the sample entropy & 0.7 & 0.8 & 0.91 & 0.86 \\
\hline
\end{tabular}

TABLE 3: Numerical range and the mean value of SE1, SE2, SE3, and SE4 sample entropy values in various gear states.

\begin{tabular}{lcccc}
\hline State of the gear & $\mathrm{G} 1$ & $\mathrm{G} 2$ & $\mathrm{G} 3$ & $\mathrm{G} 4$ \\
\hline $\begin{array}{l}\text { Numerical range of } \\
\text { SampEn1 }\end{array}$ & $0.70-0.72$ & $0.67-0.70$ & $0.72-0.75$ & $0.65-0.67$ \\
\hline $\begin{array}{l}\text { Mean value of } \\
\text { SampEn1 }\end{array}$ & 0.71 & 0.685 & 0.735 & 0.66 \\
\hline $\begin{array}{l}\text { Numerical range of } \\
\text { SampEn2 }\end{array}$ & $0.74-0.77$ & $0.77-0.8$ & $0.8-0.83$ & $0.83-0.86$ \\
\hline $\begin{array}{l}\text { Mean value of } \\
\text { SampEn2 }\end{array}$ & 0.755 & 0.785 & 0.815 & 0.845 \\
\hline $\begin{array}{l}\text { Numerical range of } \\
\text { SampEn3 }\end{array}$ & $0.79-0.85$ & $0.97-0.97$ & $0.85-0.91$ & $0.97-1.03$ \\
\hline $\begin{array}{l}\text { Mean value of } \\
\text { SampEn3 }\end{array}$ & 0.82 & 0.97 & 0.88 & 1 \\
\hline $\begin{array}{l}\text { Numerical range of } \\
\text { SampEn4 }\end{array}$ & $0.81-0.86$ & $0.91-0.96$ & $0.86-0.91$ & $0.76-0.81$ \\
\hline $\begin{array}{l}\text { Mean value of } \\
\text { SampEn4 }\end{array}$ & 0.835 & 0.935 & 0.885 & 0.785 \\
\hline
\end{tabular}

the standard vector value of gear with tooth crack is 3 , and the standard vector value of gear broken tooth is 4 . The standard vector value represents the position of the number of columns where the state of the DHNN neuron is 1 in the following binarization matrix: 1 represents the number of 1 in the first column and 2 represents the number of 1 in the second column. The data of normal, wear, crack, and broken teeth of four groups of standard gears are selected for encoding vectors, and the coding vectors is as follows:

Normal gear: $\operatorname{sim}_{-} 1=\left[\begin{array}{llll}1 & 1 & 1 & 1\end{array}\right]$.

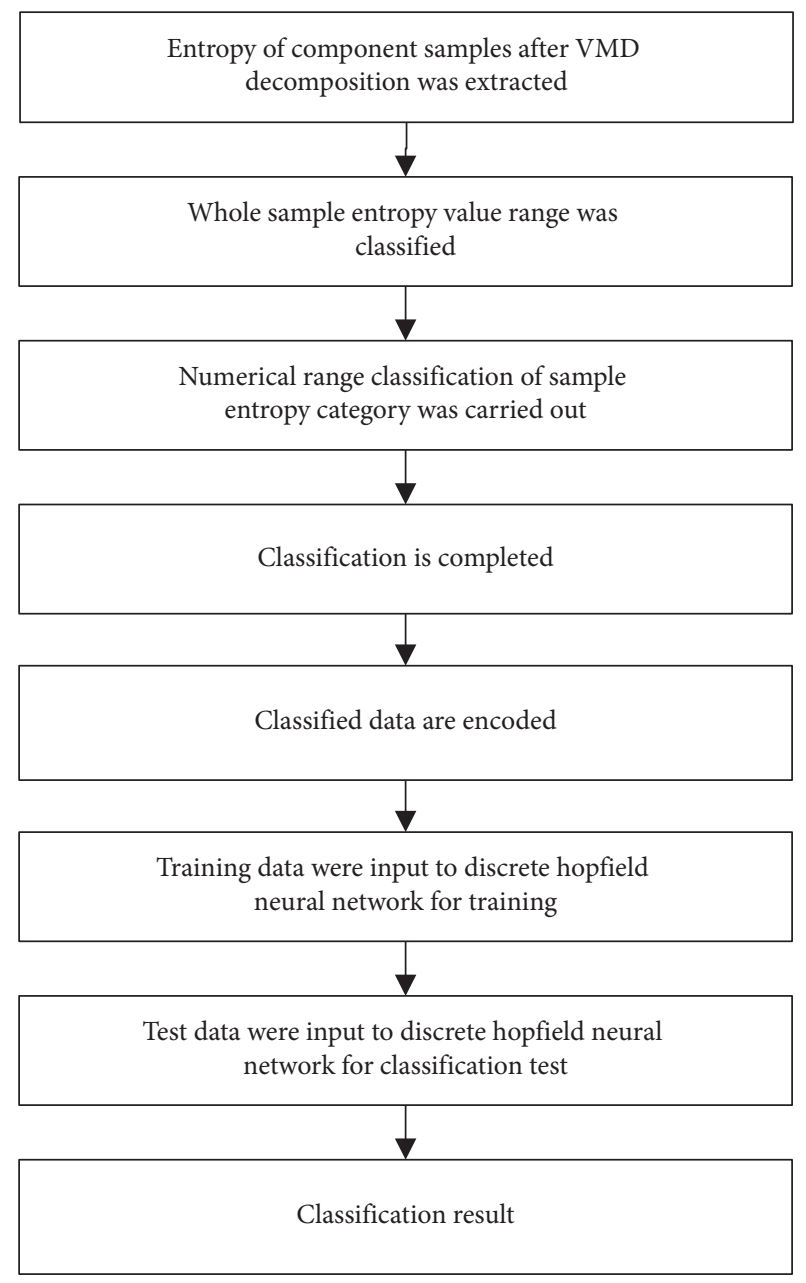

Figure 4: DHNN step diagram. 


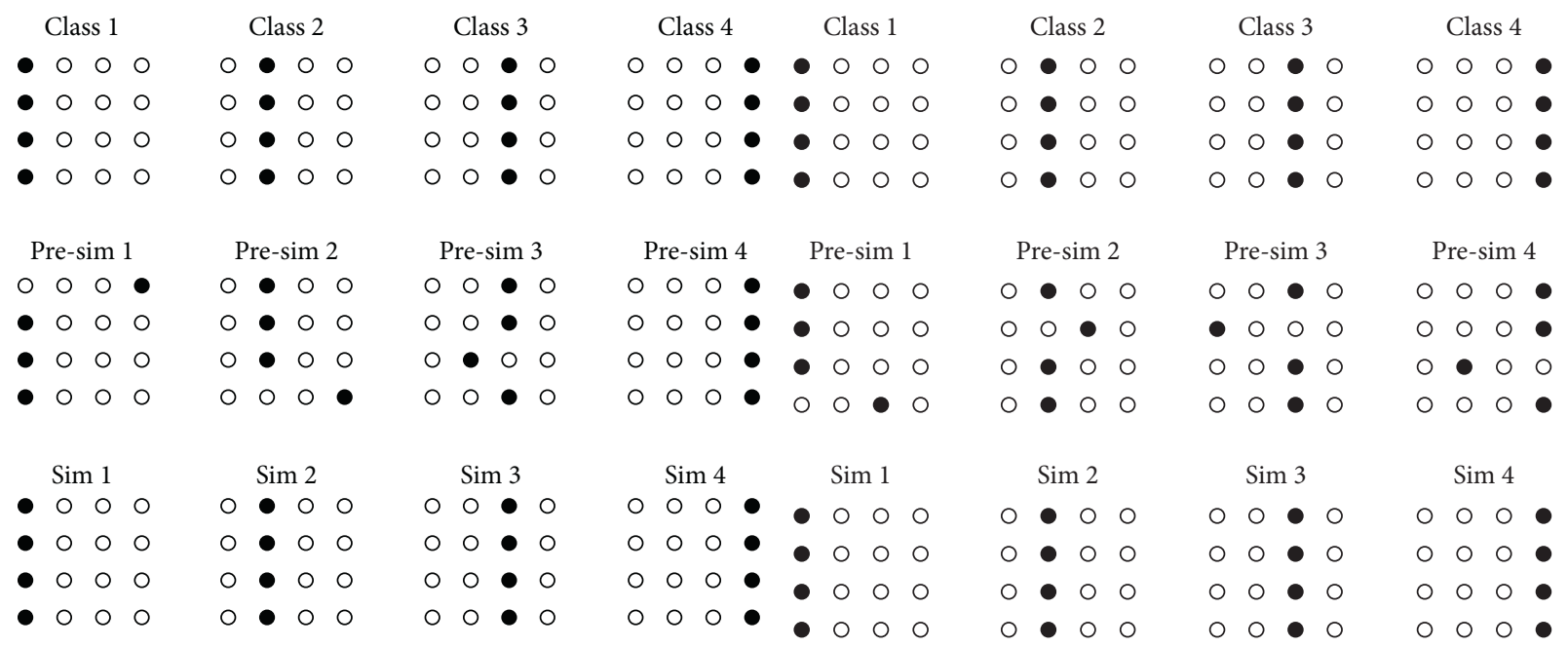

(a)

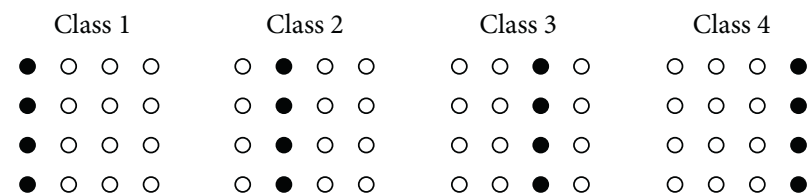

(b)

\begin{tabular}{|c|c|c|c|c|c|c|c|c|c|c|c|c|c|}
\hline \multicolumn{3}{|c|}{ Pre-sim 1} & \multicolumn{3}{|c|}{ Pre-sim 2} & \multicolumn{4}{|c|}{ Pre-sim 3} & \multicolumn{4}{|c|}{ Pre-sim 4} \\
\hline - 0 & O & 0 & O & - 0 & 0 & - & O & 0 & O & - & O & 0 & O \\
\hline - 0 & 0 & 0 & O & - 0 & 0 & O & 0 & - & 0 & O & 0 & $\bullet$ & O \\
\hline 00 & • & 0 & 0 & 00 & - & 0 & 0 & • & 0 & O & 0 & ○ & O \\
\hline - 0 & 0 & 0 & O & - 0 & 0 & O & O & - & 0 & O & 0 & ○ & O \\
\hline \multicolumn{3}{|c|}{ Sim 1} & \multicolumn{3}{|c|}{$\operatorname{Sim} 2$} & \multicolumn{4}{|c|}{ Sim 3} & \multicolumn{4}{|c|}{$\operatorname{Sim} 4$} \\
\hline - 0 & 0 & 0 & O & - 0 & 0 & 0 & O & $\bullet$ & 0 & O & 0 & • & O \\
\hline - 0 & 0 & O & O & - 0 & 0 & O & O & - & O & O & O & $\bullet$ & O \\
\hline 0 & 0 & 0 & 0 & - 0 & O & 0 & 0 & - & 0 & 0 & 0 & • & O \\
\hline - 0 & 0 & 0 & 0 & - 0 & 0 & 0 & 0 & $\bullet$ & 0 & 0 & 0 & 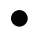 & O \\
\hline
\end{tabular}

(c)

Figure 5: CEEMDAN-DHNN gear fault diagnosis and identification results.

Gear with tooth wear: $\operatorname{sim}_{-} 2=\left[\begin{array}{llll}2 & 2 & 2 & 2\end{array}\right]$.

Gear with tooth crack: $\operatorname{sim}_{-} 3=\left[\begin{array}{llll}3 & 3 & 3 & 3\end{array}\right]$.

Gear with tooth break: sim_4 $=\left[\begin{array}{llll}4 & 4 & 4 & 4\end{array}\right]$.

Next, binarization of the coding vectors:

Normal gear:sim $1=\left[\begin{array}{llll}1 & -1 & -1 & -1 \\ 1 & -1 & -1 & -1 \\ 1 & -1 & -1 & -1 \\ 1 & -1 & -1 & -1\end{array}\right]$

Gear with tooth wear:sim_2 $=\left[\begin{array}{llll}-1 & 1 & -1 & -1 \\ -1 & 1 & -1 & -1 \\ -1 & 1 & -1 & -1 \\ -1 & 1 & -1 & -1\end{array}\right]$

$$
\begin{aligned}
\text { Gear with tooth crack:sim_3 } & =\left[\begin{array}{llll}
-1 & -1 & 1 & -1 \\
-1 & -1 & 1 & -1 \\
-1 & -1 & 1 & -1 \\
-1 & -1 & 1 & -1
\end{array}\right] \\
\text { Gear with tooth break:sim_4 } & =\left[\begin{array}{llll}
-1 & -1 & -1 & 1 \\
-1 & -1 & -1 & 1 \\
-1 & -1 & -1 & 1 \\
-1 & -1 & -1 & 1
\end{array}\right]
\end{aligned}
$$

The remaining 16 groups of data were randomly divided into three groups for gear fault status identification. Each group of data contained 4 sample entropy vectors. The following is the result of binarization of the remaining data: 


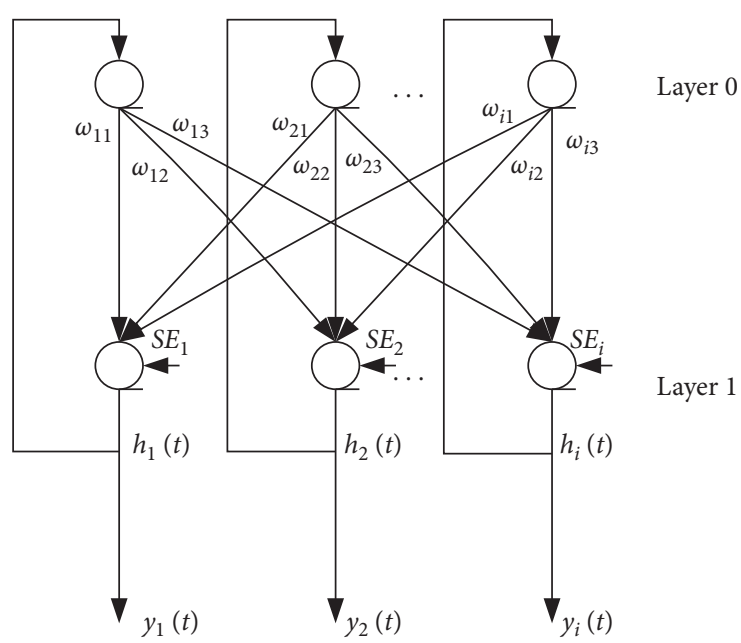

FIGURE 6: DHNN structure.

$$
\begin{aligned}
\operatorname{sim}_{-} 1 & =\left[\begin{array}{cccc}
-1 & -1 & -1 & 1 \\
1 & -1 & -1 & -1 \\
1 & -1 & -1 & -1 \\
1 & -1 & -1 & -1
\end{array}\right], \\
\operatorname{sim}_{-} 2 & =\left[\begin{array}{rrrr}
1 & -1 & -1 & -1 \\
1 & -1 & -1 & -1 \\
1 & -1 & -1 & -1 \\
-1 & -1 & 1 & -1
\end{array}\right], \\
\operatorname{sim}_{-} 3 & =\left[\begin{array}{rrrr}
1 & -1 & -1 & -1 \\
1 & -1 & -1 & -1 \\
-1 & -1 & 1 & -1 \\
1 & -1 & -1 & -1
\end{array}\right], \\
\operatorname{sim}_{-} 6 & =\left[\begin{array}{cccc}
-1 & 1 & -1 & -1 \\
-1 & 1 & -1 & -1 \\
-1 & 1 & -1 & -1 \\
-1 & 1 & -1 & -1 \\
-1 & -1 & -1 & 1 \\
-1 & 1 & -1 & 1
\end{array}\right], \\
\operatorname{sim}_{-} 5 & =\left[\begin{array}{cccc}
-1 & 1 & -1 & -1 \\
-1 & -1 & 1 & -1 \\
-1 & 1 & -1 & -1 \\
-1 & 1 & -1 & -1
\end{array}\right],
\end{aligned}
$$

$$
\begin{aligned}
& \operatorname{sim}_{-} 7=\left[\begin{array}{cccc}
-1 & -1 & 1 & -1 \\
-1 & -1 & 1 & -1 \\
-1 & 1 & -1 & -1 \\
-1 & -1 & 1 & -1
\end{array}\right] \text {, } \\
& \operatorname{sim} \_8=\left[\begin{array}{cccc}
-1 & -1 & 1 & -1 \\
1 & -1 & -1 & -1 \\
-1 & -1 & 1 & -1 \\
-1 & -1 & 1 & -1
\end{array}\right] \text {, } \\
& \operatorname{sim} \_9=\left[\begin{array}{cccc}
1 & -1 & -1 & -1 \\
-1 & -1 & 1 & -1 \\
-1 & -1 & 1 & -1 \\
-1 & -1 & 1 & -1
\end{array}\right] \text {, } \\
& \operatorname{sim}_{-} 10=\left[\begin{array}{rrrr}
-1 & -1 & -1 & 1 \\
-1 & -1 & -1 & 1 \\
-1 & -1 & -1 & 1 \\
-1 & -1 & -1 & 1
\end{array}\right] \text {, } \\
& \operatorname{sim}_{-} 11=\left[\begin{array}{cccc}
-1 & -1 & -1 & 1 \\
-1 & -1 & -1 & 1 \\
-1 & 1 & -1 & -1 \\
-1 & -1 & -1 & 1
\end{array}\right] \text {, } \\
& \operatorname{sim}_{-} 12=\left[\begin{array}{cccc}
1 & -1 & -1 & -1 \\
-1 & 1 & -1 & -1 \\
-1 & -1 & -1 & 1 \\
-1 & -1 & 1 & -1
\end{array}\right] \text {. }
\end{aligned}
$$

Selection standard of training samples of each component of the entropy, which were selected from Table 1, is 


\begin{tabular}{|c|c|c|c|c|c|c|c|}
\hline \multicolumn{4}{|c|}{ Class 1} & \multicolumn{4}{|c|}{ Class 2} \\
\hline ○ & 0 & 0 & 0 & 0 & - & O & 0 \\
\hline$\bullet$ & 0 & 0 & 0 & O & - & O & 0 \\
\hline 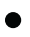 & 0 & O & 0 & O & - & 0 & 0 \\
\hline • & O & O & O & O & - & O & 0 \\
\hline \multicolumn{4}{|c|}{ Pre-sim 1} & \multicolumn{4}{|c|}{ Pre-sim 2} \\
\hline O & O & 0 & - & 0 & - & O & 0 \\
\hline 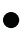 & 0 & 0 & O & 0 & - & 0 & 0 \\
\hline ? & O & 0 & 0 & 0 & $\bullet$ & 0 & 0 \\
\hline - & O & 0 & 0 & O & 0 & 0 & - \\
\hline \multicolumn{4}{|c|}{ Sim 1} & \multicolumn{4}{|c|}{$\operatorname{Sim} 2$} \\
\hline • & O & 0 & 0 & 0 & & 0 & 0 \\
\hline 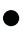 & 0 & 0 & 0 & 0 & 0 & 0 & 0 \\
\hline & O & 0 & O & 0 & & 0 & 0 \\
\hline & 0 & 0 & 0 & 0 & & 0 & 0 \\
\hline
\end{tabular}

(a)
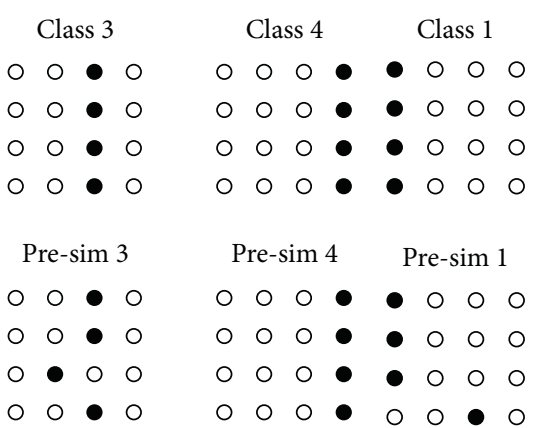

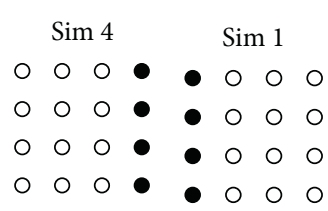

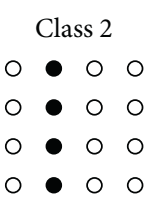

Pre-sim 2

0 - 00

$00 \cdot 0$

○ 00

$\circ-0 \quad 0$

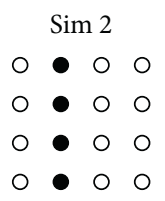

(b)

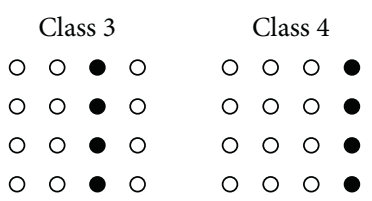

Pre-sim 3

Pre-sim 4

$00 \bullet 0$

- 000

$00 \bullet 0$

○ 0 -

000

0000

0 - 00

O 000

Sim 4

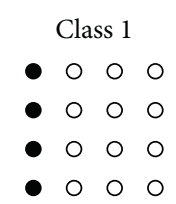

\begin{tabular}{cccc}
\multicolumn{3}{c}{ Pre-sim 1} \\
- & 0 & 0 & 0 \\
- & 0 & 0 & 0 \\
0 & 0 & 0 & 0 \\
- & 0 & 0 & 0
\end{tabular}

Sim 1

- 000

- 000

- 000

- 000

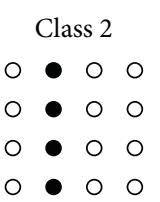

Pre-sim 2

0 - 00

- 00

○ 000

- 00

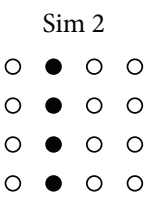

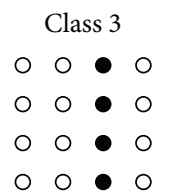

Class 4

O 00

000

O 00

O 00
O 000

000

$\circ 00$

○ 000

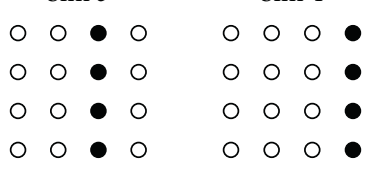

)

\begin{tabular}{|c|c|c|c|c|c|c|}
\hline \multicolumn{4}{|c|}{ Pre-sim 3} & \multicolumn{3}{|c|}{ Pre-sim 4} \\
\hline$\bullet$ & 0 & 0 & 0 & • & 0 & 00 \\
\hline O & 0 & - & 0 & 0 & - & 00 \\
\hline 0 & 0 & - & 0 & 0 & 0 & 0 \\
\hline 0 & 0 & - & 0 & 0 & O & - 0 \\
\hline \multicolumn{4}{|c|}{ Sim 3} & \multicolumn{3}{|c|}{$\operatorname{Sim} 4$} \\
\hline O & 0 & - & 0 & 0 & 0 & 00 \\
\hline 0 & 0 & - & 0 & 0 & 0 & 00 \\
\hline 0 & 0 & - & 0 & 0 & 0 & 00 \\
\hline 0 & 0 & - & 0 & 0 & 0 & 0 \\
\hline
\end{tabular}

(c)

FIGURE 7: VMD-DHNN gear fault diagnosis and identification results.

the sample entropy of the signal sequence SampEn3 normal gear, the gear with tooth wear and tear of sample entropy SampEn2 signal sequence, the gear with tooth crack sample entropy SampEn3 signal sequence, and the gear tooth broken sample entropy SampEn1 input signal sequence to the DHNN training, after waiting for neural network training, and then, the remaining 12 group gears as the unknown state data were input into the trained neural network to identify the state. Before the sample entropy value is input to the DHNN, the standard data group is firstly binary coded, and then, the coding vectors are input to the DHNN for training.

\section{Gear Fault Diagnosis Based on the VMD Sample Entropy and DHNN}

The 16 sets of sample entropy value data were binarized and input to DHNN for network training as a standard sample. Then, the remaining 12 sets of sample entropy values were tested as the test data group. The above binarization matrix was input into DHNN for classification and identification, and the VMD-DHNN recognition results are shown in Figures $7(\mathrm{a})-7(\mathrm{c})$.

Among them, class1 represents normal gear standard data, class 2 represents gear with tooth wear standard data, class 3 represents gear with tooth crack standard data, and class 4 represents gear with tooth break standard data. Pre$\operatorname{sim} 1$ represents the first test data, pre-sim 2 represents the second test data, pre-sim 3 represents the third test data, and pre-sim 4 represents the fourth test data. Sim 1 represents the first test data classification result, $\operatorname{sim} 2$ represents the second test data classification result, sim 3 represents the third test data classification result, and $\operatorname{sim} 4$ represents the fourth test data classification result.

As shown in Figures 7(a)-7(c), 11 of the 12 test samples of the DHNN model classifier correspond to the fault features, and one of them is not recognized due to the unclear features but is not recognized incorrectly. 


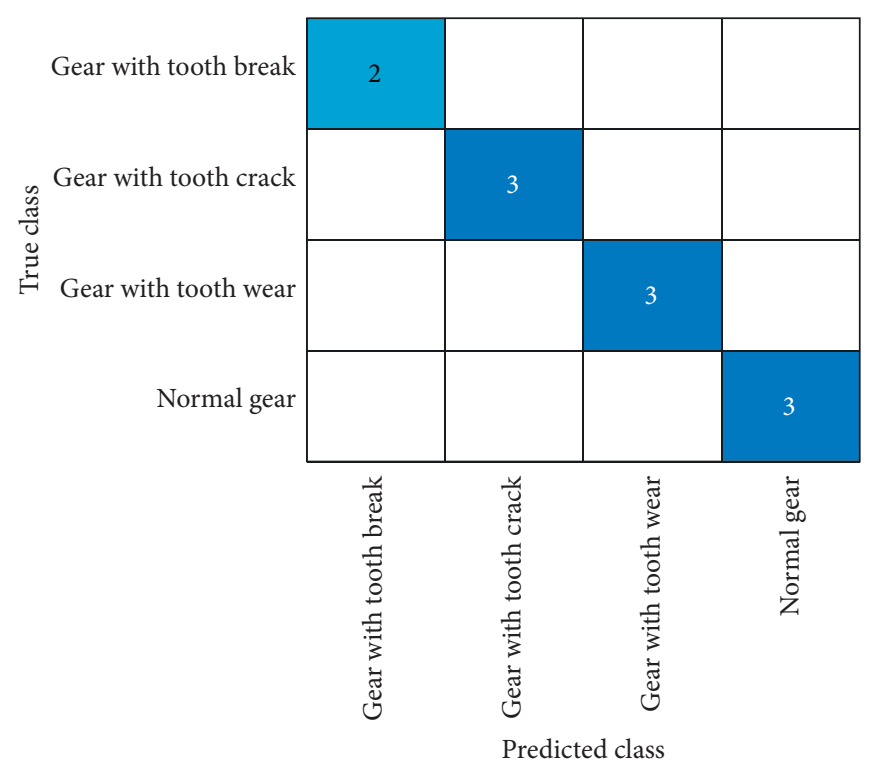

FIGURE 8: Confusion matrix of the VMD-DHNN classification results.

In order to get the accuracy of gear fault identification more clearly, the confusion matrix of VMD-DHNN classification results is made below. According to Figure 8, normal gear, gear with tooth wear, and gear with tooth crack faults are correctly classified, and there is a classification error in the gear with tooth break fault. However, the failure classification of gear with tooth break fault is not identified as other gear fault types.

In order to verify the superiority of the VMD-DHNN algorithm, the VMD-DHNN algorithm is compared with complete ensemble empirical mode decomposition adaptive noise (CEEMDAN)-DHNN algorithm and empirical mode decomposition (EMD)-DHNN algorithm in the accuracy of gear fault diagnosis. Firstly, the training gear fault feature vectors are input into CEEMDAN-DHNN and EMD-DHNN to obtain the gear fault identification result. Then, the test gear fault feature vectors are input into the trained CEEMDAN-DHNN and EMD-DHNN, and the classification result of gear fault is obtained.

The CEEMDAN-DHNN recognition results are shown in Figures 5(a)-5(c).
As shown in Figure 5(c), the gear with tooth break fault is identified as gear with tooth crack fault. The best recognition rate of $91.67 \%$ was obtained using CEEMDAN-DHNN.

In order to get the accuracy of gear fault identification more clearly, the confusion matrix of CEEMDAN-DHNN classification results is made below. According to Figure 9, normal gear, gear with tooth wear, and gear with tooth crack faults are correctly classified, and there is a classification error in the gear with tooth break fault.

The EMD-DHNN recognition results are shown in Figures 10(a)-10(c). It can be seen from Figure 10(c) that the gear with tooth break fault is identified as gear with tooth crack fault, the gear with tooth wear fault is identified as normal gear, and the gear with tooth crack fault is identified as the gear with tooth wear fault. The best recognition rate of $75 \%$ was obtained using EMD-DHNN.

In order to get the accuracy of gear fault identification more clearly, the confusion matrix of EMD-DHNN classification results is given in Figure 11.

Table 4 shows the gear fault diagnosis accuracy of different signal decomposition algorithms combined with DHNN and SVM. 


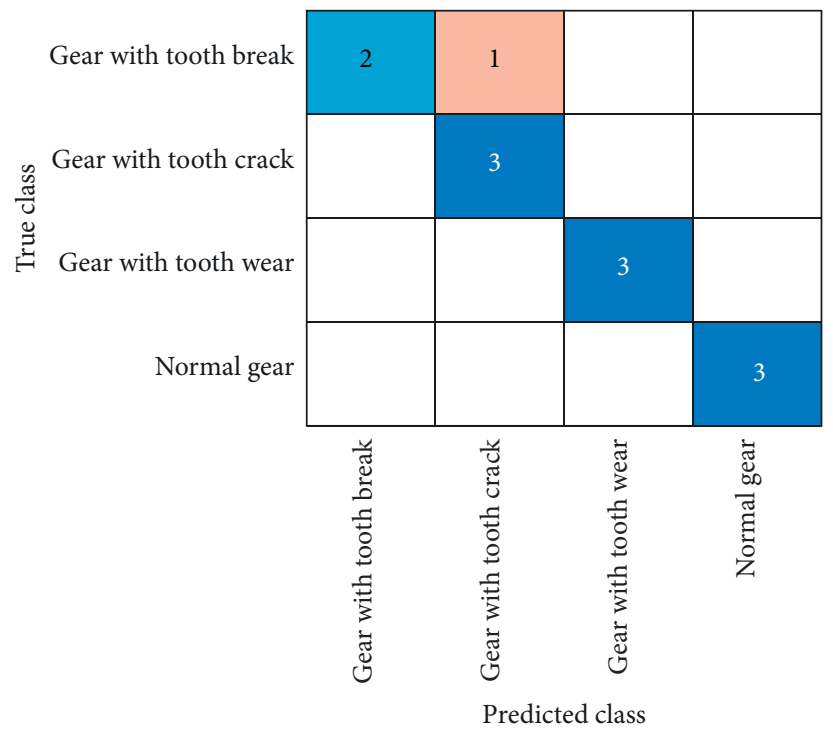

FIgure 9: Confusion matrix of the CEEMDAN-DHNN classification results.

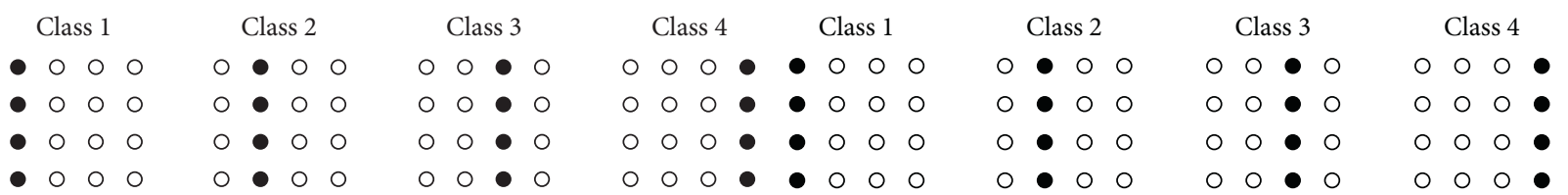

\begin{tabular}{|c|c|c|c|c|c|c|c|c|c|c|c|c|c|c|c|c|c|c|c|c|c|c|c|c|c|c|c|}
\hline \multicolumn{3}{|c|}{ Pre-sim 1} & \multicolumn{3}{|c|}{ Pre-sim 2} & \multicolumn{4}{|c|}{ Pre-sim 3} & \multicolumn{4}{|c|}{ Pre-sim 4} & \multicolumn{4}{|c|}{ Pre-sim 1} & \multicolumn{3}{|c|}{ Pre-sim 2} & \multicolumn{4}{|c|}{ Pre-sim 3} & \multicolumn{3}{|c|}{ Pre-sim 4} \\
\hline 00 & 0 & - & o & - 0 & o & O & $\circ$ & • & 0 & o & 0 & O & $\bullet$ & • & o & O & o & O & - 0 & O & O & O & - & O & o & O & O \\
\hline - 0 & O & 0 & 0 & - 0 & 0 & 0 & O & $\bullet$ & O & O & 0 & O & $\bullet$ & $\bullet$ & O & o & o & 0 & 0 & o & $\bullet$ & o & o & O & o & 0 & O \\
\hline - 0 & O & ० & ० & - 0 & 0 & 0 & $\bullet$ & O & 0 & O & 0 & O & $\bullet$ & • & O & O & 0 & O & - 0 & O & o & ○ & - & O & o & • & O \\
\hline - 0 & O & 0 & O & 00 & $\bullet$ & O & O & $\bullet$ & O & 0 & O & O & $\bullet$ & 0 & 0 & $\bullet$ & 0 & 0 & - 0 & 0 & 0 & O & $\bullet$ & O & O & O & O \\
\hline \multicolumn{3}{|c|}{ Sim 1} & \multicolumn{3}{|c|}{$\operatorname{Sim} 2$} & \multicolumn{4}{|c|}{$\operatorname{Sim} 3$} & \multicolumn{4}{|c|}{$\operatorname{Sim} 4$} & \multicolumn{4}{|c|}{ Sim 1} & \multicolumn{3}{|c|}{$\operatorname{Sim} 2$} & \multicolumn{4}{|c|}{$\operatorname{Sim} 3$} & \multicolumn{3}{|c|}{$\operatorname{Sim} 4$} \\
\hline - 0 & O & 0 & ○ & - 0 & 0 & 0 & O & $\bullet$ & 0 & O & O & O & $\bullet$ & - & 0 & O & 0 & 0 & - 0 & 0 & O & $\circ$ & & O & O & ○ & \\
\hline - 0 & O & 0 & O & - 0 & 0 & 0 & O & $\bullet$ & 0 & 0 & 0 & O & $\bullet$ & $\bullet$ & 0 & o & 0 & 0 & - 0 & 0 & 0 & 0 & - & 0 & 0 & 0 & O \\
\hline - 0 & 0 & 0 & O & - 0 & 0 & 0 & 0 & $\bullet$ & 0 & 0 & 0 & 0 & $\bullet$ & & 0 & 0 & 0 & 0 & - 0 & 0 & 0 & 0 & - & 0 & 0 & 0 & o \\
\hline - 0 & 0 & 0 & 0 & - 0 & 0 & 0 & 0 & - & 0 & 0 & 0 & O & & & 0 & 0 & 0 & 0 & - 0 & 0 & 0 & 0 & & 0 & 0 & 0 & o \\
\hline
\end{tabular}

(a)

(b)

\begin{tabular}{|c|c|c|c|c|c|c|c|c|c|c|c|}
\hline \multicolumn{3}{|c|}{ Class 1} & \multicolumn{3}{|c|}{ Class 2} & \multicolumn{3}{|c|}{ Class 3} & \multicolumn{3}{|c|}{ Class 4} \\
\hline 0 & 0 & 0 & 0 & - 0 & 0 & 0 & 0 & - 0 & 0 & 0 & 0 \\
\hline 0 & 0 & 0 & 0 & - 0 & 0 & 0 & 0 & - 0 & 0 & 0 & 0 \\
\hline 0 & 0 & 0 & 0 & - 0 & 0 & 0 & 0 & - 0 & 0 & 0 & 0 \\
\hline 0 & 0 & 0 & 0 & - 0 & 0 & 0 & 0 & - 0 & 0 & 0 & 0 \\
\hline
\end{tabular}

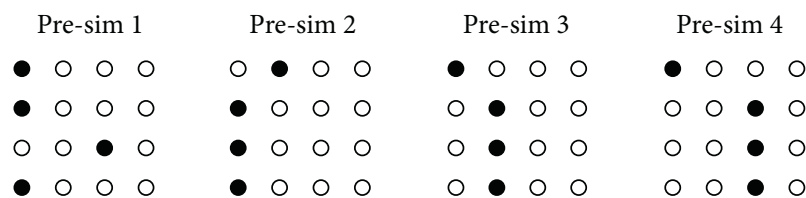

\begin{tabular}{|c|c|c|c|c|c|c|c|c|c|c|}
\hline & & & $\mathrm{Sir}$ & $\mathrm{m} 2$ & & & $\operatorname{Sim} 3$ & & & Sim \\
\hline 0 & 0 & 0 & - 0 & 0 & 0 & 0 & - 0 & 0 & 0 & 0 \\
\hline O & 0 & 0 & - 0 & 0 & 0 & 0 & - 0 & 0 & 0 & 0 \\
\hline 0 & 0 & 0 & - 0 & 0 & 0 & 0 & - 0 & 0 & 0 & 0 \\
\hline & 0 & 0 & 0 & 0 & 0 & 0 & - 0 & 0 & 0 & 0 \\
\hline
\end{tabular}

(c)

Figure 10: EMD-DHNN gear fault diagnosis and identification results. 


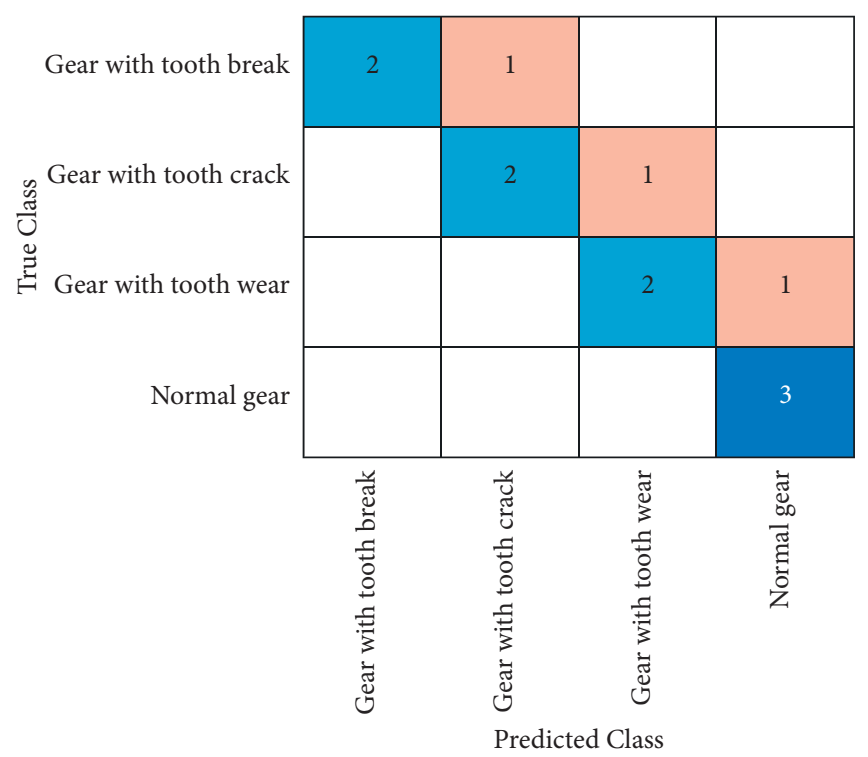

FIgURE 11: Confusion matrix of the EMD-DHNN classification results.

TABle 4: Gear fault diagnosis accuracy of different signal decomposition algorithms combined with DHNN.

\begin{tabular}{lcc}
\hline Signal decomposition algorithm & Classification algorithm & Accuracy (\%) \\
\hline EMD & DHNN & 75 \\
CEEMDAN & DHNN & 91.67 \\
VMD & DHNN & 91.67 \\
EMD & SVM & 50 \\
CEEMDAN & SVM & 66.67 \\
VMD & SVM & 75 \\
\hline
\end{tabular}

\section{Conclusions}

In this paper, a VMD-DHNN method is proposed and applied to nonstationary signal decomposition. Firstly, the vibration signals of nonstationary gear fault are decomposed by the VMD, and their center frequencies are accurately separated. Then, each decomposed IMF is an extracted sample entropy value, and the extracted feature value is formed into the feature vector. Finally, the gear fault feature vector is input to DHNN for fault diagnosis.

The optimized VMD algorithm can avoid the disadvantages of the EMD algorithm, and VMD has the advantages of fast operation speed and high-frequency accuracy of separated IMFs center. The DHNN has the advantage of associative memory and can further shorten the operation time. The combination of VMD-DHNN can improve the fault diagnosis time of gear and reduce the damage.

The VMD-DHNN is more accurate in identifying faults such as normal gear, gear with tooth wear, and gear with tooth crack. This method can be further applied to defect detection in additive manufacturing and welding. Moreover, the intelligent optimization algorithm can be utilized to optimize the parameters of VMD so that it can be adaptive to decompose the signal.

The limitation of this article is that we need to encode the signal in advance and then diagnose the fault in the input to DHNN, which may increase the complexity of the method.

\section{Data Availability}

The data used to support the findings of this study are available from the corresponding author upon request.

\section{Conflicts of Interest}

The authors declare that there are no conflicts of interest regarding the publication of this paper.

\section{Acknowledgments}

This work was supported by the National Natural Science Foundation of China (nos. 51875195 and 52075163).

\section{References}

[1] B. Liu, S. Riemenschneider, and Y. Xu, "Gearbox fault diagnosis using empirical mode decomposition and Hilbert spectrum," Mechanical Systems and Signal Processing, vol. 20, no. 3, pp. 718-734, 2006.

[2] D. Bianchi, E. Mayrhofer, M. Gröschl, G. Betz, and A. Vernes, "Wavelet packet transform for detection of single events in acoustic emission signals," Mechanical Systems and Signal Processing, vol. 64-65, pp. 441-451, 2015.

[3] O. D. Mohammed, M. Rantatalo, J.-O. Aidanpää, and U. Kumar, "Vibration signal analysis for gear fault diagnosis 
with various crack progression scenarios," Mechanical Systems and Signal Processing, vol. 41, no. 1-2, pp. 176-195, 2013.

[4] J. Rafiee and P. W. Tse, "Use of autocorrelation of wavelet coefficients for fault diagnosis," Mechanical Systems and Signal Processing, vol. 23, no. 5, pp. 1554-1572, 2009.

[5] Y. Tian, J. Ma, C. Lu, and Z. Wang, "Rolling bearing fault diagnosis under variable conditions using LMD-SVD and extreme learning machine," Mechanism and Machine Theory, vol. 90, pp. 175-186, 2015.

[6] X. Liu, L. Bo, and H. Luo, "Bearing faults diagnostics based on hybrid LS-SVM and EMD method," Measurement, vol. 59, pp. 145-166, 2015.

[7] M. Buzzoni, E. Mucchi, G. D’Elia, and G. Dalpiaz, “Diagnosis of localized faults in multistage gearboxes: a vibrational approach by means of automatic EMD-based algorithm," Shock and Vibration, vol. 2017, Article ID 8345704, 22 pages, 2017.

[8] J. Rafiee, M. A. Rafiee, and P. W. Tse, "Application of mother wavelet functions for automatic gear and bearing fault diagnosis," Expert Systems with Applications, vol. 37, no. 6, pp. 4568-4579, 2010.

[9] X. Hu, S. Peng, and W.-L. Hwang, "EMD revisited: a new understanding of the envelope and resolving the modemixing problem in AM-FM signals," IEEE Transactions on Signal Processing, vol. 60, no. 3, pp. 1075-1086, 2011.

[10] Y. Li, X. Liang, Y. Yang, M. Xu, and W. Huang, "Early fault diagnosis of rotating machinery by combining differential rational spline-based LMD and K-L divergence," IEEE Transactions on Instrumentation and Measurement, vol. 66, no. 11, pp. 3077-3090, 2017.

[11] K. Dragomiretskiy and D. Zosso, "Variational mode decomposition," IEEE Transactions on Signal Processing, vol. 62, no. 3, pp. 531-544, 2013.

[12] J. Ding, D. Xiao, and X. Li, "Gear fault diagnosis based on genetic mutation particle swarm optimization VMD and probabilistic neural network algorithm," IEEE Access, vol. 8, pp. 18456-18474, 2020 .

[13] J. Ding, L. Huang, D. Xiao, and X. Li, "GMPSO-VMD algorithm and its application to rolling bearing fault feature extraction," Sensors, vol. 20, no. 7, 1946 pages, 2020.

[14] J. Li, X. Yao, H. Wang, and J. Zhang, "Periodic impulses extraction based on improved adaptive VMD and sparse code shrinkage denoising and its application in rotating machinery fault diagnosis," Mechanical Systems and Signal Processing, vol. 126, pp. 568-589, 2019.

[15] X. Yan and M. Jia, "Application of CSA-VMD and optimal scale morphological slice bispectrum in enhancing outer race fault detection of rolling element bearings," Mechanical Systems and Signal Processing, vol. 122, pp. 56-86, 2019.

[16] X. Zhang, Q. Miao, H. Zhang, and L. Wang, "A parameteradaptive VMD method based on grasshopper optimization algorithm to analyze vibration signals from rotating machinery," Mechanical Systems and Signal Processing, vol. 108, pp. 58-72, 2018.

[17] Y. Li, X. Wang, Z. Liu, X. Liang, and S. Si, "The entropy algorithm and its variants in the fault diagnosis of rotating machinery: a review," IEEE Access, vol. 6, pp. 66723-66741, 2018.

[18] Y. Chen, T. Zhang, Z. Luo, and K. Sun, "A novel rolling bearing fault diagnosis and severity analysis method," Applied Sciences, vol. 9, no. 11, p. 2356, 2019.

[19] Y. Chen, T. Zhang, W. Zhao, Z. Luo, and H. Lin, "Rotating machinery fault diagnosis based on improved multiscale Amplitude-aware permutation entropy and multiclass relevance vector machine," Sensors, vol. 19, no. 20, p. 4542, 2019.
[20] C. Liu, L. Zhu, and C. Ni, "Chatter detection in milling process based on VMD and energy entropy," Mechanical Systems and Signal Processing, vol. 105, pp. 169-182, 2018.

[21] Z. Zhang, H. Li, G. Meng, X. Tu, and C. Cheng, "Chatter detection in milling process based on the energy entropy of VMD and WPD," International Journal of Machine Tools and Manufacture, vol. 108, pp. 106-112, 2016.

[22] A. A. Abdoos, P. Khorshidian Mianaei, and M. Rayatpanah Ghadikolaei, "Combined VMD-SVM based feature selection method for classification of power quality events," Applied Soft Computing, vol. 38, pp. 637-646, 2016.

[23] W. Deng, H. Liu, J. Xu, H. Zhao, and Y. Song, "An improved quantum-inspired differential evolution algorithm for deep belief network," IEEE Transactions on Instrumentation and Measurement, vol. 69, no. 10, pp. 7319-7327, 2020.

[24] G. Qiu, Y. Gu, and Q. Cai, “A deep convolutional neural networks model for intelligent fault diagnosis of a gearbox under different operational conditions," Measurement, vol. 145, pp. 94-107, 2019.

[25] J. Lei, C. Liu, and D. Jiang, "Fault diagnosis of wind turbine based on Long Short-term memory networks," Renewable Energy, vol. 133, pp. 422-432, 2019.

[26] Z. Chen and W. Li, "Multisensor feature fusion for bearing fault diagnosis using sparse autoencoder and deep belief network," IEEE Transactions on Instrumentation and Measurement, vol. 66, no. 7, pp. 1693-1702, 2017.

[27] H. Shao, H. Jiang, Y. Lin, and X. Li, "A novel method for intelligent fault diagnosis of rolling bearings using ensemble deep auto-encoders," Mechanical Systems and Signal Processing, vol. 102, pp. 278-297, 2018.

[28] S. Cofre-Martel, P. Kobrich, E. Lopez Droguett, and V. Meruane, "Deep convolutional neural network-based structural damage localization and quantification using transmissibility data," Shock and Vibration, vol. 2019, Article ID 9859281, 27 pages, 2019.

[29] J. J. Hopfield, "Neural networks and physical systems with emergent collective computational abilities," Proceedings of the National Academy of Sciences, vol. 79, no. 8, pp. 25542558, 1982.

[30] D. Xiao, J. Ding, X. Li, and L. Huang, "Gear fault diagnosis based on kurtosis criterion VMD and SOM neural network," Applied Sciences, vol. 9, no. 24, p. 5424, 2019. 